\title{
Design of a flow controlled asymmetric droplet splitter using computational fluid dynamics
}

Bert Verbruggen ${ }^{1}$, Tamara Tóth ${ }^{1}$, Yegermal Tesfaw Atalay ${ }^{1}$, Frederik Ceyssens ${ }^{2}$, Pieter Verboven ${ }^{1}$, Robert Puers ${ }^{2}$, Bart Nicolai ${ }^{1}$, Jeroen Lammertyn ${ }^{1 *}$

${ }^{1}$ BIOSYST-MeBioS, University of Leuven

Willem de Croylaan 42, 3001 Leuven, Belgium

${ }^{2}$ ESAT-MICAS, University of Leuven

Kasteelpark Arenberg 10, 3001 Leuven, Belgium

*Corresponding author

Tel: +32 16321452

Fax: +3216322955

E-mail: jeroen.lammertyn@biw.kuleuven.be

\section{Abstract:}

In this work the design of a segmented flow microfluidic device is presented that allows droplet splitting ratios from 1:1 up to $20: 1$. This ratio can be dynamically changed on chip by altering an additional oil flow. The design was fabricated in PDMS chips using the standard SU-8 mold technique and does not require any valves, membranes, optics or electronics. To avoid a trial and error approach, fabricating and testing several designs, a computational fluid dynamics (CFD) model was developed and validated for droplet formation and splitting. The model was used to choose between several variations of the splitting T-junction with the extra oil inlet, as well to predict the additional flow rate needed to split the droplets in various ratios. Experimental and simulated results were in line, suggesting the model's suitability to optimize future designs and concepts. The resulting asymmetric droplet splitter design opens possibilities for controlled sampling and improved magnetic separation in bio-assay applications.

\section{Keywords}

Asymmetric Splitting, Droplets in Micro-channels, Segmented Flow Microfluidics, Computational Fluid Dynamics 


\section{Introduction}

Recently, segmented microfluidic flow has become popular because of its specific benefits over continuous microfluidics. The confined volumes of droplets or plugs prevent cross contamination (Song and Ismagilov 2003), require less volume of reagents in general, and allow for droplets to be slowed or stopped for incubation (Huebner et al. 2009). The high throughput possibilities of segmented flow (Anna et al. 2003) enable, for example, the mass production of particles (Nisisako and Torii 2008) or quantum dots (Nightingale and De Mello 2010), the study of protein crystallization (Zheng et al. 2004) and parallelized cell cultivation (Grodrian et al. 2004). More recent applications of segmented flow are the detection of single molecules (Srisa-Art et al. 2010) or DNA mutations (Pekin et al. 2011), quantification of protein expression (Huebner et al. 2007) and enzymatic assays (Huebner et al. 2008). Production of chemical or biological compounds with segmented flow has been demonstrated by Song et al. (2003) and Günther and Jensen (2006) but has yet to be adopted for industrial implementation. To further enhance and stimulate the development and use of microfluidic systems outside research labs, more standardization, parallelization and scalable production methods are needed. A transition from elastomer channels to glass, plastics, silicon and steel has already started to accommodate the needs of large scale production, and all microfluidic features developed in research should therefore preferably be material independent, easily producible and robust (Whitesides 2006).

Segmented flow includes several unit operations, such as droplet formation (Anna et al. 2003; Thorsen et al. 2001), droplet merging (Christopher et al. 2009; Niu et al. 2008) and droplet splitting (Link et al. 2004). Equal droplet splitting is used to parallelize reactions (Grodrian et al. 2004) or to separate particles (Lombardi and Dittrich 2011). The latter is useful for the integration of bio-assays on microfluidic chips (Pan et al. 2011). However, improvements to the droplet splitting systems are needed before bio-assays can be fully implemented in segmented flow microfluidics. Song et al. (2003) and Link et al. (2004) designed a splitting Tjunction with a longer and a shorter narrow arm, creating a different flow resistance in the two branches and thus a different pressure drop. The channels reconnect further downstream, effectively equalizing the pressure at that point, thus avoiding unstable systems. Nie and Kennedy (2010) refined the unequal splitting, adding pillars to the T-junction and loop system and achieving splitting ratios up to $34: 1$. This allows sampling 
from droplets at different stages of a complex assay with minimal impact on the droplet volume. It also becomes possible to remove more than half of the original droplet volume from magnetic beads during an assay, greatly improving the efficiency of the system. After three sequential equal spits, only $87.50 \%$ is removed. In the case of a 10:1 splitting, three repetitions would remove $99.9 \%$ of the original volume. In these designs, the splitting ratio is fixed for one chip, causing a lack of flexibility during the optimization of a bio-assay. Changes such as decreasing the original droplet aspect ratio, increasing the sampling volume or increasing the concentration of particles would lead to repeated fabrication of new chips with different splitting ratios. Alternatively, computational fluid dynamics (CFD) models can be used to design the microfluidic chip. De Menech et al. (2008) and Sivasamy et al. (2011) used CFD to identify distinct droplet formation mechanisms (i.e. squeezing, dripping and jetting) in a T-junction. Recently Liu and Zhang (2011) also used a numerical model to study droplet formation in microfluidic cross-junctions. Yamada et al. (2008) presented the idea of an asymmetric droplet splitter, using an additional oil flow to split pL droplets at $290 \mathrm{~Hz}$ to produce microparticles resulting in a split ratio of $4: 1$.

The aim of this article is to develop a splitting system on a channel based microfluidic chip that can split droplets reliably in unequal parts, at low flow rates, ranging from nearly equal splitting up to $20: 1$, with the help of CFD modeling (Atalay et al. 2009; Atalay et al. 2008) and to validate the design afterwards. Figure 1 (a) gives an overview of the used design: starting from the regular T-junction for droplet formation (position 1) and the narrow split and loop system of Nie and Kennedy (2010) (2 and 3), the additional oil inlet of Yamada et al. (2008) is added to regulate the pressure drop and thus the droplet split ratio (4, 5 or 6$)$. While the concept is proven based on an implementation in PDMS, it can readily be extended to other materials including glass, silicon and steel as it does not rely on the elastic properties of PDMS, nor does it use membranes, valves, lasers or electronics. Obviously the materials' hydrophobicity should be accounted for or changed using a coating (Dreyfus et al. 2003). 


\subsection{Reagents}

HFE-7500 fluorocarbon oil (3M, Zwijndrecht, Belgium) was used as the carrier or continuous phase and water as a discreet or sample phase. The oil contained $1 \mathrm{w} / \mathrm{w} \%$ of a custom made PFPE-PEG-PFPE surfactant (Holtze et al. 2008) kindly provided by the Weitz lab at Harvard University, USA and had a viscosity $(\mu)$ of $0.00124 \mathrm{~Pa} \cdot \mathrm{S}$ and a density $(\rho)$ of $1614 \mathrm{~kg} / \mathrm{m}^{3}$. The surface tension $(\gamma)$ between oil and water was $0.0035 \mathrm{~N} / \mathrm{m}$. In order to visualize the droplets, $2.28 \mathrm{mg} / \mathrm{l}$ of fluorescein sodium salt (Sigma-Aldrich, Bornem, Belgium) was added to the water. All fluids were filtered using $0.22 \mu \mathrm{m}$ filters (Millipore, Billerica, MA, USA) before injection into the channels.

\subsection{Chip design and fabrication}

The chip design is shown in figure 1 . The channel dimensions, except the extra oil inlet, were kept constant for all experiments. All channels were $60 \mu \mathrm{m}$ high. The width of the main channel was $200 \mu \mathrm{m}$ while the water inlet was $100 \mu \mathrm{m}$ wide. Under the right flow conditions the water formed droplets in the oil spontaneously at the first T-junction (1). At the splitting zone (2) the main channel narrowed down to $50 \mu \mathrm{m}$, elongating the droplets. At the second T-junction the droplets were split in two daughter droplets. After the split the two branches broadened back to $100 \mu \mathrm{m}$, making the droplets move slower and thus improving the imaging results. To avoid a different pressure build-up in the two branches of the split, they were reconnected (3), to allow the oil to flow from one branch to the other, effectively equalizing the pressure. A line of pillars (50 $\mu \mathrm{m})$ prevented the droplets from changing channels. In this work an additional oil inlet $(50 \mu \mathrm{m})$ was added to the lower branch of the loop. Using CFD simulations, the effect of three different locations $(4,5$ and 6$)$ on the splitting performance was studied. The geometry was varied as well: a straight inlet (4), an opposing inlet (b) and a parallel inlet (c). The best design was fabricated and tested experimentally. All designs were drawn in AutoCAD (Autodesk Inc., San Rafael, CA, USA) and plotted on high density photomasks (Koenen, Ottobrunn, Germany). 
(a)

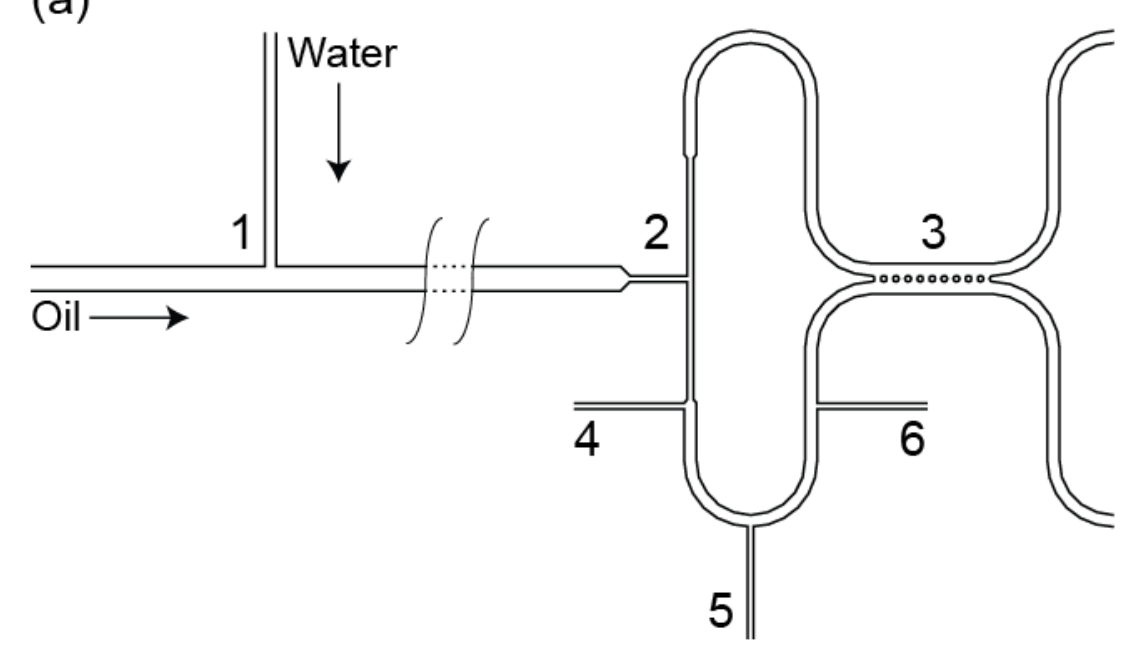
hydrophobic and thus improve the droplet formation and stability. r (b) - - - ר

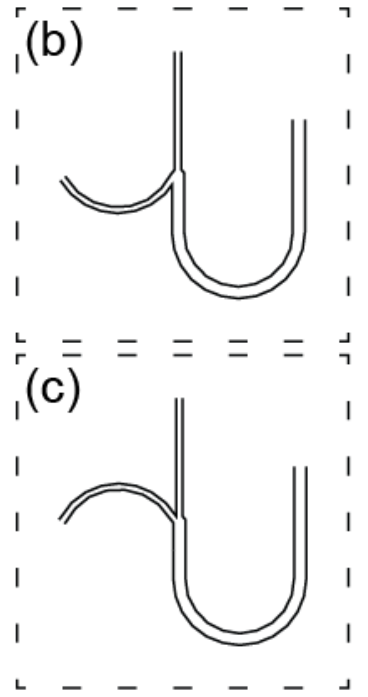

The microfluidic chips were fabricated in polydimethylsiloxane (PDMS) using softlithographic techniques as described by Duffy et al. (1998). A layer of SU-8 photoresist (Microchem, Newton, MA, USA) was spin-coated on 3 inch wafers at 2000 RPM and softbaked at $65^{\circ} \mathrm{C}$ for $20 \mathrm{~min}$. The wafer was then exposed to UV-light (20 $\mathrm{mJ} / \mathrm{cm}^{2}$ ) through the photomask and baked again at $65^{\circ} \mathrm{C}$ for 20 min. Finally the SU-8 was developed and hardbaked at $95^{\circ} \mathrm{C}$ for $1 \mathrm{~h}$. The resulting molds had straight angled features and a uniform height of $60 \mu \mathrm{m}$, as determined with a surface profilometer (Dektak 3030, Veeco, NY, USA). To produce the PDMS chip the monomer and the curing agent (Dow Corning, Midland, MI, USA) were mixed, degassed and cast on the SU-8 mold. After $4 \mathrm{~h}$ at $65{ }^{\circ} \mathrm{C}$ the PDMS became solid and could be pealed from the mold. Holes for the tubing were pierced and the channels were closed by sealing the PDMS slab to a glass slide using oxygen plasma. Finally the channels were coated with Aquapel (Pittsburgh Glass Works LLC, Pittsburgh, PA, USA) to make them more

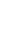

Fig 1: (a) Design of the microfluidic channels (not to scale). The water and oil flows meet in the T-junction (1), droplets form spontaneously and are pushed to the right. In the narrow splitting T-junction (2) the droplets split and each daughter droplet follows a different branch of the loop. At the pillars (3), the oil can pass through to equilibrate pressure variations, but the droplets remain separated. Using CFD simulations, three locations $(4,5$ or 6$)$ for the extra inlet were compared. (b) The design of the opposing inlet and (c) the design of the parallel inlet. 


\subsection{Experimental setup}

The microfluidic chip was mounted on the stage of an inverted fluorescence microscope (IX-71, Olympus, Tokyo, Japan) and the inlets of the chip were connected to glass syringes (Hamilton, Bonaduz, Switzerland) by PEEK tubes (IDEX, Wertheim-Mondfeld, Germany). PHD 2000 syringe pumps (Harvard Apparatus, Holliston, MA, USA) were used to precisely control the flow rate in the channels. Oil flow rates varied between 0.1 and $10 \mu \mathrm{L} / \mathrm{min}$ and water flow rates between 0.01 and $5 \mu \mathrm{L} / \mathrm{min}$. The fluorescent signal, of the fluorescein in the water, was detected by an electron multiplier charge coupled density (EM CCD) camera (C9100-13, Hamamatsu, Shizuoka, Japan) mounted on the microscope and the resulting images were recorded for later analysis. During the experiments it was observed that changing the flow rate of the fluids did not have an instantaneous effect on the droplet aspect ratio. A transition period of 10 to 20 seconds was needed for the droplets to reach the predicted droplet aspect ratio. To remove this effect from the results, droplets were recorded 1 minute after changing the flow rates.

\subsection{Data analysis}

The experimental results were analyzed with a custom MATLAB script (The Mathworks, Natick, MA) to determine the 2-D projection of the droplets in the channel. Frame by frame the whole recording was scanned for droplets and their size and position were calculated. First the average intensity in all frames was measured to determine a threshold and the images were subsequently binarized. The edge of the projections of the droplets was then determined independently of fluorescence variations or location on the image and used to calculate the projected surface and volume of the droplet, assuming rectangular channels. The dimensionless droplet aspect ratio, droplet length over droplet width, was used, which allows a comparison over different channel dimensions and thus between different studies (Garstecki et al. 2006).

All measurement errors due to inhomogeneous illumination or rounding in the MATLAB edge detection procedure were found to be below $1 \%$ and smaller than the natural droplet variations during formation ( $2-$ 4\%). At very high flow rates ( $>30 \mu \mathrm{L} / \mathrm{min}$ ) the script started to overestimate the projected surface of the droplet and thus the droplet volume, because of blurry images, but all the flow rates used in this work were below this value. 


\section{The multiphase model}

\subsection{Numerical simulation}

Segmented flow microfluidics is a multiphase flow phenomenon using two immiscible liquids and therefore the interface between these fluids can be tracked using the volume of fluid (VOF) modeling method, one of the most widely applied methods in modeling of free surfaces. Hereby, a single set of momentum equations is shared by the fluids, and the volume fraction of each of the fluids is tracked, in each computational cell throughout the domain. The momentum and continuity equation used to model the transport of fluids in a channel are:

$$
\begin{gathered}
\frac{\partial \rho}{\partial t}+\nabla \cdot(\rho \mathbf{u})=0(E q \cdot 1) \\
\frac{\partial(\rho \mathbf{u})}{\partial t}+\nabla \cdot(\rho \mathbf{u u})=-\nabla p+\nabla \cdot\left[\mu\left(\nabla \mathbf{u}+\nabla \mathbf{u}^{T}\right)\right]+\rho \mathbf{g}+\mathbf{F}_{S F}(E q \cdot 2)
\end{gathered}
$$

where $\rho\left(\mathrm{kg} / \mathrm{m}^{3}\right)$ and $\mu(\mathrm{Pa} \cdot \mathrm{s})$ are the discontinuous fluid density and viscosity respectively, $\mathbf{u}(\mathrm{m} / \mathrm{s})$ is the velocity vector, $p(\mathrm{~Pa})$ is the pressure, $\boldsymbol{g}\left(\mathrm{m} / \mathrm{s}^{2}\right)$ is gravitational acceleration vector and $\mathbf{F}_{\mathbf{S F}}(\mathrm{N} / \mathrm{m})$ is the surface tension force.

The VOF method is a fixed-mesh method and the interface between immiscible fluids is modeled as a discontinuity in the characteristic function such as the volume fraction. The volume fraction indicates the fluid amount within the control volume, while the interface between the phases is tracked by the solution of continuity equation for the volume fraction $\alpha$ of the water phase:

$$
\frac{\partial \alpha}{\partial t}+\mathbf{u} \cdot \nabla \alpha=0(E q .3)
$$

\section{The value of $\alpha$ in a cell ranges between 1 and 0 , where $\alpha=1$ represents a cell which is completely filled with} water, while $\alpha=0$ represents a cell which is completely filled with oil and $0<\alpha<1$ represents the interface 
between oil and water. The material properties in the transport equations are computed as weighted averages based on the volume fraction of the individual fluid in a single computational cell:

$$
\begin{aligned}
& \rho=\rho_{2}+\left(\rho_{1}-\rho_{2}\right) \alpha(E q .4) \\
& \mu=\mu_{2}+\left(\mu_{1}-\mu_{2}\right) \alpha(E q .5)
\end{aligned}
$$

151 where the subscripts 1 and 2 denote water and oil, respectively. The effects of surface tension, wall adhesion and other dominant forces in the flow channel are also accounted for in the VOF model (Hirt and Nichols 1981). It is assumed that the velocity field is continuous across the interface but there is a pressure jump at the interface due to the presence of surface tension. This pressure droplet across the interface depends on the surface tension coefficient between water and oil $\sigma(\mathrm{N} / \mathrm{m})$ and the surface curvature $\kappa$ and can be modeled using the continuum surface force (CSF) model of Brackbill et al. (1992):

$$
\mathbf{F}_{S F}=\frac{\sigma \rho \kappa}{\frac{1}{2}\left(\rho_{1}+\rho_{2}\right)} \nabla \alpha(E q .6)
$$

With $\kappa=\nabla \cdot \widehat{\mathbf{n}}=\nabla \cdot\left(\frac{\mathbf{n}}{|\mathbf{n}|}\right)(E q .7)$

where $\mathbf{n}$ is the normal vector to the interface surface calculated from $\mathbf{n}=\nabla \alpha$ and $\widehat{\mathbf{n}}$ is the unit normal vector. The surface normal is evaluated in interface-containing grid cells and requires knowledge of the amount of volume of fluid present in the cell.

The wetting properties of the fluid-wall interface are extremely important in determining flow patterns (Dreyfus et al. 2003). Ordered flow patterns can be obtained when the continuous phase completely wets the microchannels. The hydrophobicity or hydrophilicity of a solid surface can be expressed quantitatively using contact angles. The effects of the wall adhesion can be estimated easily within the CSF surface tension model in terms of the contact angle between the fluid and wall $\left(\theta_{w}\right)$. describe flows at the micrometer scale: 


$$
\mathrm{Ca}=\frac{\mu \bar{u}_{x}}{\sigma}(E q .9)
$$

With $\mu(P a \cdot s)$ is the viscosity, $\overline{\mathrm{u}}_{\mathrm{x}}(\mathrm{m} / \mathrm{s})$ the average velocity of oil in the flow direction and $\sigma(\mathrm{N} / \mathrm{m})$ the surface tension. The capillary number can be used to replace average velocity or total flow rate, in order to compare different systems and channel dimensions. For example, a flow rate of $0.5 \mu \mathrm{L} / \mathrm{min}$ in the main channel has a speed of $6.94 \times 10^{-4} \mathrm{~m} / \mathrm{s}$ and thus a Ca of $2.47 \times 10^{-5}$. The same flow rate in the narrow Tjunction has a speed of $2.78 \times 10^{-3} \mathrm{~m} / \mathrm{s}$, increasing the Ca four times to $9.87 \times 10^{-5}$.

\subsection{Numerical solution procedure}

The unsteady fluid flow equations were solved using a pressure-based solver and explicit scheme-based VOF method, which was developed for the multiphase simulation in FLUENT 13 (Ansys Inc., USA). The pressure implicit with splitting of operators (PISO) algorithm was used in the transient calculations to obtain the pressure-velocity coupling. The pressure discretization was obtained by using the PRESTO (pressure staggering option) method and the second-order upwind method was used for spatial discretization of the momentum equation. For high accuracy calculation of the interface position in the cell the geometric reconstruction method (based on piecewise linear interface construction or PLIC) was applied. The volume fraction equation was solved using first-order implicit time discretization method. The convergence criteria for the solution of continuity and momentum equations were set in the order of $10^{-6}$ and a time step ranging from 1 to $10 \mu$ sec.

\subsection{Mesh sensitivity analysis}

To reduce the computational time it is important to choose a mesh with the lowest number of finite volume elements, while still providing sufficient accuracy. Numerical simulations were executed at different mesh densities. With refined meshes the interface computation was more accurate, whereas coarse meshes resulted in numerical diffusion of the interface. This leads to underestimation of the size of the droplets calculated from coarse mesh simulations. For instance, there was less than $1 \%$ difference in droplet ratio between a mesh size of $4 \mu \mathrm{m}$ and $5 \mu \mathrm{m}$, while the droplet aspect ratio decreased by $7.5 \%$ when its size increased to $10 \mu \mathrm{m}$. A visual comparison is shown in figure 2. However, calculation with a fine mesh requires 


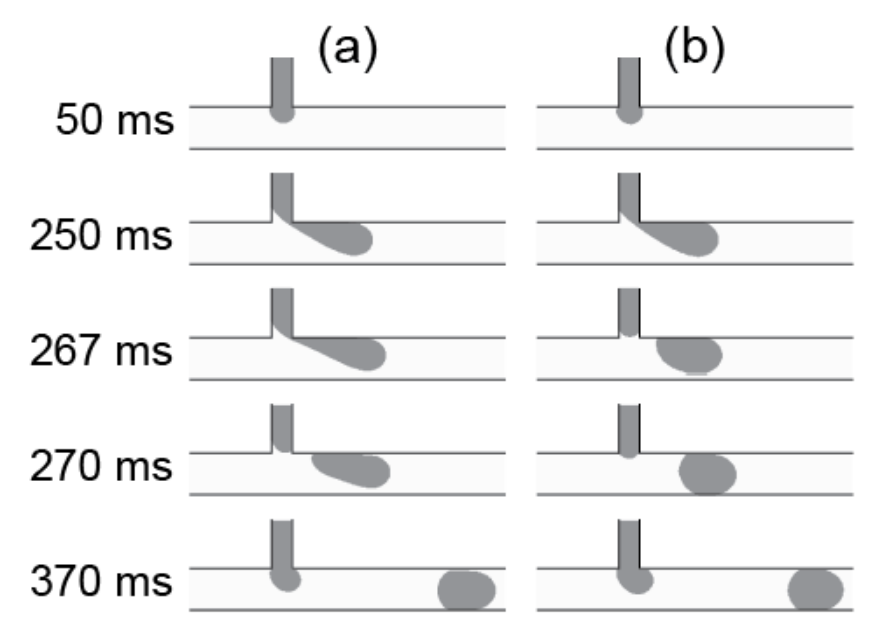

Fig 2: The effect of different mesh sizes during the simulation of droplet formation in a T-junction. With a mesh size of $5 \mu \mathrm{m}$ (a), the droplets pinched off between 267 and $270 \mathrm{~ms}$. Using a coarser mesh of $10 \mu \mathrm{m}$ (b) resulted in droplet pinched off before $267 \mathrm{~ms}$. This small difference already caused a 7.5\% decrease of the aspect ratio.

\subsection{Experimental validation using droplet formation}

198 In the T-junction design the local flow field is determined by the channel geometry, the flow rates and the 199 physical properties of the fluids and the channel wall. De Menech et al. (2008) described three types of 200 droplet formation in the T-junction: 'squeezing', 'dripping' and 'jetting', which are following each other at 201 increasing volumetric flow rate of oil $\left(Q_{\text {oil }}\right)$ and thus increasing $\mathrm{Ca}$ (figure 3). Squeezing takes place at $\mathrm{Ca}<$ 0.015 ( $a$ and b). With increasing $\mathrm{Ca}$, shear stresses start playing a larger role in droplet formation leading to 203 the dripping regime at $\mathrm{Ca}>0.015$ (c). Jetting needs an even higher Ca, which was not simulated. In order to 204 work with a slow and precise droplet formation only the squeezing regime was used for the following 205 simulations and experiments. 
(a)

(b)

(c)

Fig 3: Simulation results for droplet formation with $Q_{\text {water }}=0.4 \mu \mathrm{L} / \mathrm{min}$ and $Q_{o i l}$ of (a) $0.6 \mu \mathrm{L} / \mathrm{min}$, (b) $4.8 \mu \mathrm{L} / \mathrm{min}$, (c)

$66 \mu \mathrm{L} / \mathrm{min}$. (a) and (b) are examples of the squeezing mechanism, with droplets touching both channel walls (Ca <

0.015), (c) shows the dripping mechanism, here the drop is smaller than the channel.

At the squeezing regime the interfacial forces dominate over shear stress and the breakup is mainly triggered by the pressure droplet across the growing droplet at the channel intersection (Garstecki et al. 2006). This can be illustrated based on numerical simulation results shown in figure 4 . Small flow rates were used to improve the visualization of the breakup: $Q_{o i l}$ is $0.8 \mu \mathrm{L} / \mathrm{min}$ and $Q_{\text {water }}$ is $0.1 \mu \mathrm{L} / \mathrm{min}$. During the droplet formation in a T-junction system the aqueous phase penetrates into the main channel and the droplet starts to grow (a). The droplet is distorted in the downstream direction due to the flow in the main channel and the pressure gradient (b). The droplet continues to grow and at the same time the interface on the upstream side of the droplet moves downstream (c). The flow of the continuous phase is confined to a thin wetting film between the droplet and the walls of the channel, resulting in a build-up of pressure upstream in the main channel. When the interface approaches the downstream edge of the inlet of the aqueous phase, the neck that connects the water inlet channel with the droplet will elongate and eventually break (d). Once the droplet detaches from the continuous phase the pressure in the main channel decrease again and the cycle repeats itself. It is clear from figure 4 that the simulated results $(a-d)$ qualitatively agree very well with the experimental results $(e-h)$. 

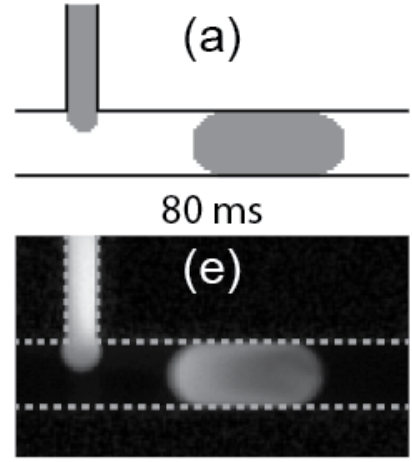
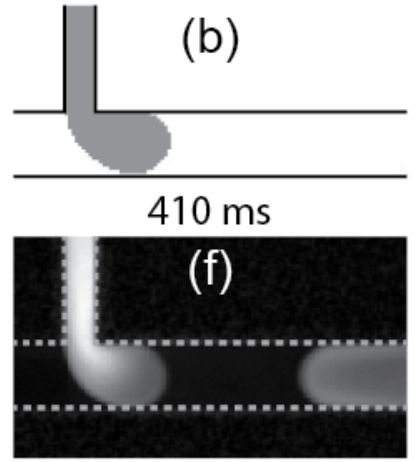
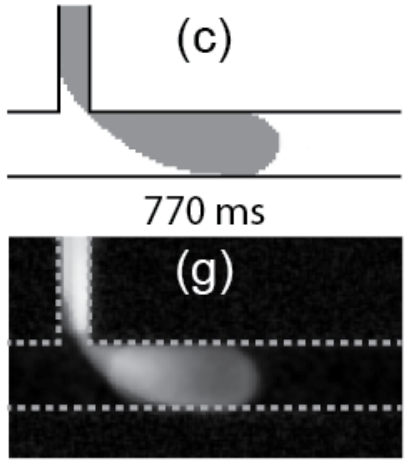

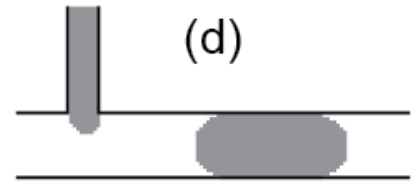

$890 \mathrm{~ms}$

(h)

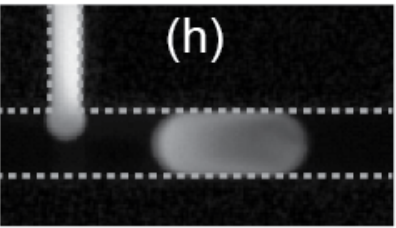

Fig 4: Simulated results of droplet formation (a $-d)$ and corresponding experimental results $(e-h) . Q_{o i l}=0.8 \mu L / m i n$

and $Q_{\text {water }}=0.1 \mu \mathrm{L} / \mathrm{min}$. Non-uniformity in fluorescent intensity is caused by the flow gradient in the inlet channel and

bleaching: fluid in the center of the channel flows faster and thus remains more intense. Inside the droplet the intensity becomes homogenous in seconds.

To further validate the developed numerical model the simulated values were compared quantitatively with the experimental droplet aspect ratios in a specific T-junction design, for a constant $Q_{\text {oil }}$ of $0.6 \mu \mathrm{L} / \mathrm{min}$. Figure 5 shows the relation between the droplet aspect ratio and the ratio of the volumetric flow rates, defined by $Q_{\text {water }} / Q_{\text {oil }}$. At lower $Q_{\text {water }} / Q_{\text {oil }}$ the droplet aspect ratio goes to 1 , representing a nearly round droplet, which is still touching the channel walls. At higher $Q_{\text {water }} / Q_{\text {oill }}$ the relation becomes linear. These experimental results are in good agreement with those reported by (Garstecki et al. 2006). The graph shows that the simulated and the experimental data follow the same trend over the whole range, yet at low $Q_{\text {water }} / Q_{\text {oil }}$ the simulation overestimates the aspect ratio significantly, while underestimating at higher $Q_{\text {water }} / Q_{\text {oil }}$. This cannot be explained by the uncertainties on the experiments and while this discrepancy was not further studied at this point, it can probably be explained by the reduced complexity of the pseudo 3D simulation. Bringing into account the slight slope of the channel wall and the slightly rounded corners might improve the fit, but would greatly increase the computational cost. Also the flows inside the droplet and of the oil around the droplet were not simulated for this reason (Kolb and Cerro 1993; de Lózar et al. 2008; Olbricht 1996). 


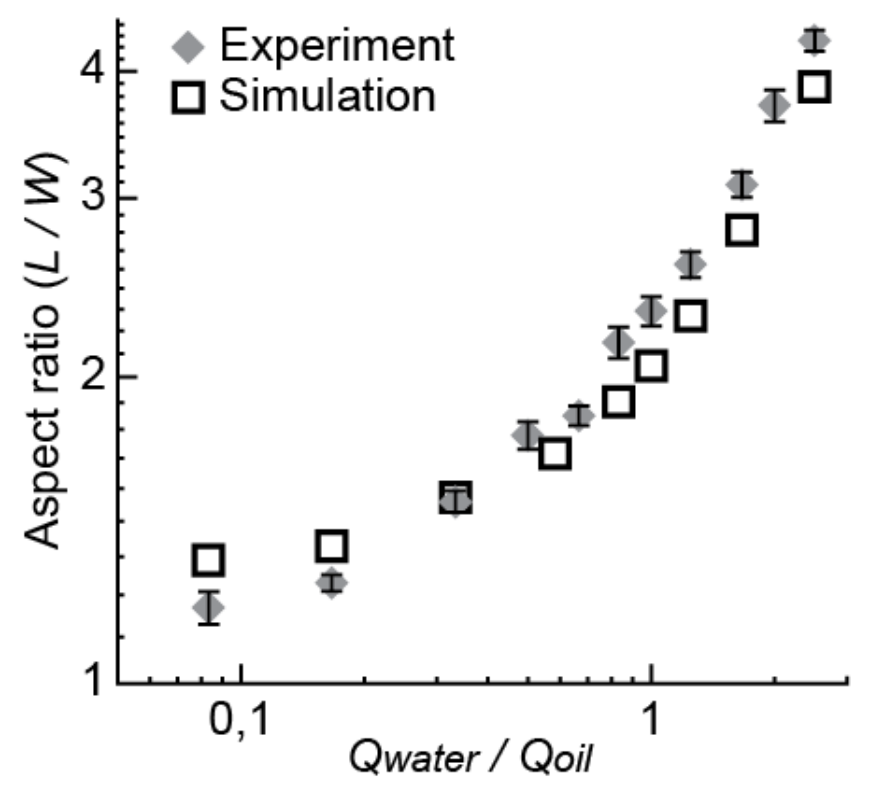

Fig 5: Droplet aspect ratio $(L / W)$ versus $Q_{\text {water }} / Q_{\text {oil }}$ diagram for experimental and numerically simulated data, $Q_{o i l}=0.6$ $\mu \mathrm{L} / \mathrm{min}$.

\section{Design of the flow controlled unequal splitter}

\subsection{Design of T-junction split}

To study the parameters that govern the process of droplet splitting at the T-junction, a range of different flow rates and initial droplet aspect ratios was simulated. Figure 6 (a) shows the result of a longer droplet (aspect ratio $=7.2$ ) at a flow rate of $50.68 \mu \mathrm{L} / \mathrm{min}$, corresponding to a $\mathrm{Ca}$ of 0.01 . When the droplet arrives at the split, it bulges into the two branches and blocks both of them (i). The droplet is then stretched and squeezed by the carrier fluid flowing from the though main channel (ii). In this process a neck with a circular shape forms in the central part of the junction. This neck thins out until breakup occurs and two daughter droplets are formed (iii). Finally, the detached droplets relax to a channel filling ellipsoid-like shape. Experiments confirm the simulations (b). For a more typically sized droplet (aspect ratio $=1.6$ ) at the same operational conditions, the simulation (c) shows that the droplet goes into either of the branches without breakup. In order to break this droplet, the capillary number must be increased by increasing the flow rate tenfold. The corresponding experiment (d) has the same end result, although a small discrepancy remains during the transition time (ii). 


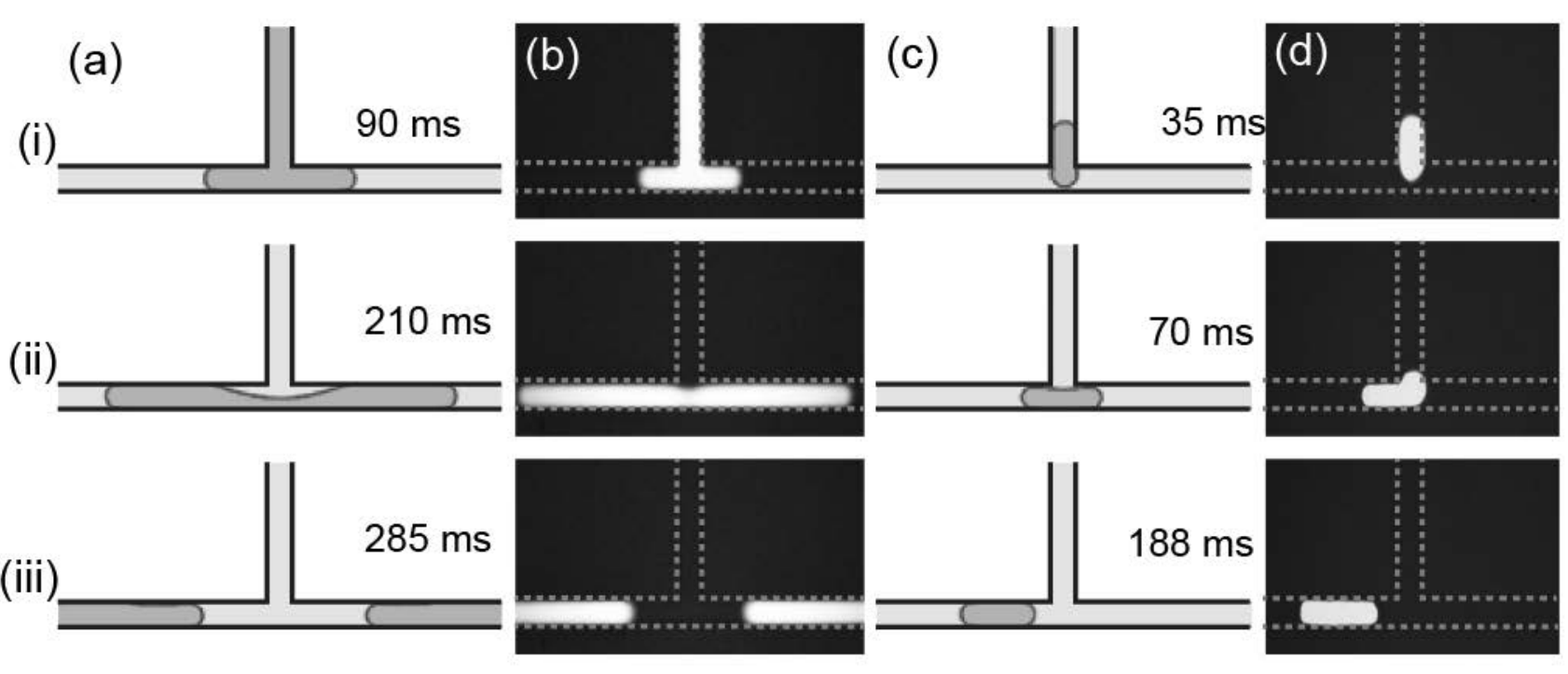

Fig 6: Visual comparison of simulated and experimental results of droplet splitting in a T-junction at $\mathrm{Ca}=0.01$ or a flow

rate of $50.68 \mu \mathrm{L} / \mathrm{min}:(\mathrm{a})$ and (b) a large droplet with aspect ratio $=7.2,(\mathrm{c})$ and (d) a smaller droplet with aspect ratio $=$

\subsection{Channel height is $60 \mu \mathrm{m}$ and channel width $200 \mu \mathrm{m}$.}

Figure 6 shows breaking and non-breaking conditions of both simulated and experimental data. It can be seen

that the smaller droplets split only at high $\mathrm{Ca}$ and thus high flow rates, while the larger droplets break up at lower flow rates. The critical capillary number in function of the droplet elongation, as derived by Link et al.

(2004) is $C a_{\text {crit }}=D \epsilon_{0}\left(\frac{1}{\epsilon_{0}^{2 / 3}}-1\right)^{2}$ with $\epsilon_{0}=\frac{L}{\pi W} . D$ was fitted as 1 for their system, depending on viscosity,

surface tension and system geometries. When taking into account the difference in viscosity and surface tension, $D=0.22$ for the system used in figure 6 and this model is plotted as dotted line in figure 7 . As can be seen, the model and data don't match and actually no $D$ can exist to fit this data perfectly as the analytical model will always predicts droplet breakup at $\epsilon_{0}=1$ or $\frac{L}{W}=\pi$, while both the numerically simulated and the 271 experimental data have non-breaking droplets at higher aspect ratios. This can indicate our system falls out of

272 the valid range of the analytic model, probably due to some of the assumptions made when deriving the analytical model, e.g. square channel cross-sections. Both the analytical model and our numerical model are approximations based on certain assumptions and both can thus predict slightly different results. 

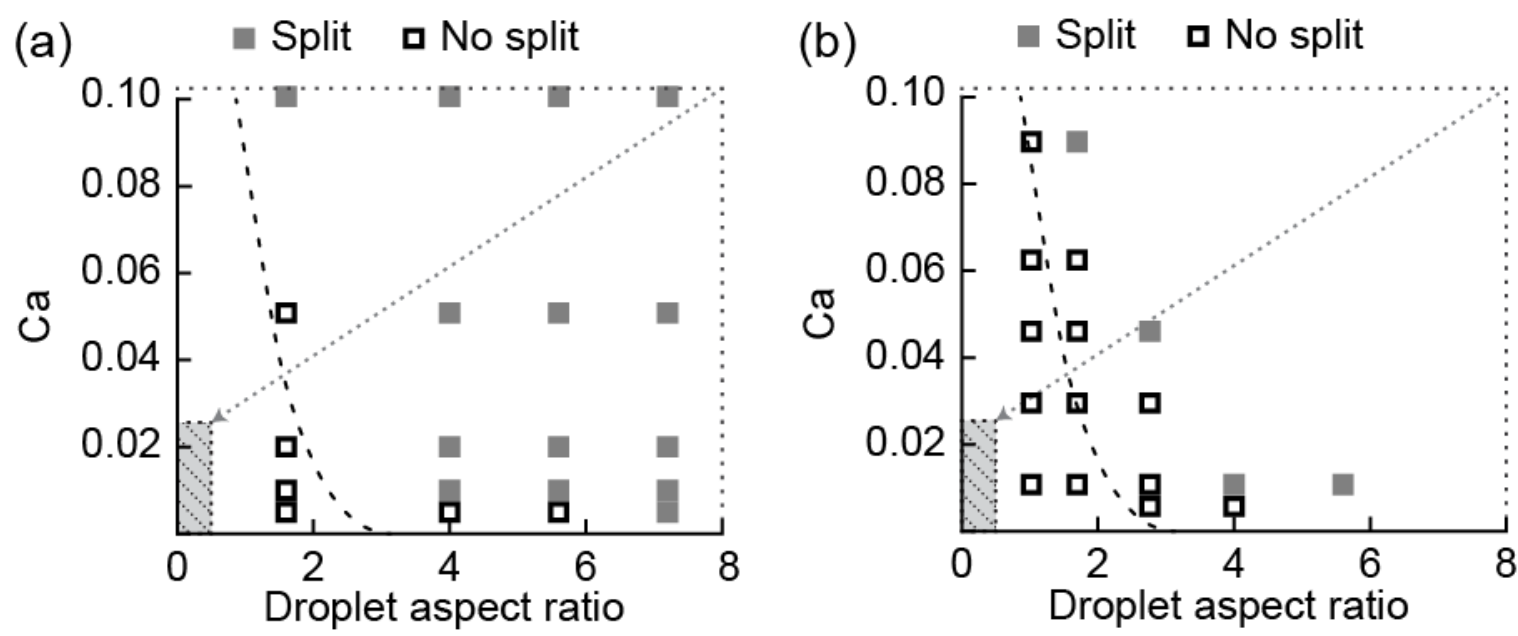

275

Fig 7: (a) Split or no split diagram based on simulated results (b) experimental results. The dotted curve indicates the analytical model of Link et al. (2004), using $D=0.22$. When using the narrow T-junction, the tested whole range is reduced to the small hatched area.

A lower flow rate was preferred to prevent high pressures and to limit the blurry droplet edges during imaging. Therefore the channel was narrowed, locally creating a higher $\mathrm{Ca}$ and increasing the aspect ratio of the droplet. Decreasing the width of the channel at the split from $200 \mu \mathrm{m}$ to $50 \mu \mathrm{m}$ locally increased the average velocity 4 times and the droplet aspect ratio $(L / W) 16$ times which allowed the splitting of much smaller droplets at low flow rates. If the droplets used in figure 7 would be split in the narrow T-junction, with the locally increased elongation and $\mathrm{Ca}$, all would break up. Otherwise, to create non-breaking droplets very small aspect ratios and Ca would be needed. Al tested conditions are reduced to the small hatched area, representing the maximal condition range with non-breaking droplets. As no droplets with an aspect ratio smaller than 1 can be formed in our system, every droplet formed will be split.

\subsection{Design of the extra inlet}

To make an asymmetric splitting system, able to split droplets in different ratios, the pressure in the two branches must be controlled with operational parameters only. This was achieved by the addition of an additional oil flow to one of the branches, which could be controlled independently from the oil and water flow at the formation junction. Flow rates can be changed dynamically and thus the splitting ratio can also be varied on one design, without complicating the production process with valves. Developing an efficient microfluidic system capable of splitting droplets in different volume ratios by trial-and-error would require 
numerous designs. As described above models were developed to simulate the equal droplet splitting in a Tjunction. The same approach was followed to predict the effect of an extra flow of oil through the additional inlet and thus reduce the amount of fabrication work. A design is proposed with the regular T-junction for droplet formation, the narrow split and loop system of Nie and Kennedy (2010) and the additional oil inlet of Yamada et al. (2008) to regulate the pressure droplet and thus the droplet split ratio (figure 1). Three locations for the inlet perpendicular on the loop were simulated (figure 1 (a)): close to the inlet (4), in the middle of the loop (5) or near the end (6). During these simulations $Q_{\text {oill }}, Q_{\text {water }}$ and $Q_{\text {extra }}$ were equal to 6 $\mu \mathrm{L} / \mathrm{min}$. The three systems had an impact on the splitting ratio, but the difference was small. For position 1,2 and 3 the splitting ratios were 1.6:1, 1.6:1 and 1.5:1, respectively. The inlets closer to the split had a slightly larger impact on the splitting ratio and position 1 was therefore used in all following designs. Next the effect of the geometry of the inlet at position 4 was investigated and a parallel (figure $1(a)$ ), a perpendicular (b) and an opposite (c) inlet were compared using the same flow rates as above. Both the inlet parallel to the main flow direction as the inlet opposite to the flow direction had a splitting ratio of 1.9:1, but in case of the opposing inlet but the system was unstable and the small daughter droplet was split a second time at the extra oil inlet. The perpendicular inlet showed a split ratio of 1.6:1. From these results it can be concluded that the design with a parallel inlet close to the splitting zone was the best design, resulting in the most stable splitting ratio. It was fabricated and used for all following simulations and experiments.

\subsection{Simulating and validating the flow of the extra inlet}

In the previous simulations, the highest splitting ratio was not yet sufficient. To reach higher splitting ratios, the $Q_{\text {extra }} / Q_{o i l}$ had to be increased. This was achieved by increasing $Q_{\text {extra }}$ or by decreasing $Q_{o i l}$. To avoid the imaging problems at high flow rates, $Q_{\text {water }}$ and $Q_{\text {oil }}$ were reduced to $0.06 \mu \mathrm{L} / \mathrm{min}$ and $0.6 \mu \mathrm{L} / \mathrm{min}$ respectively. With these flow rates, the droplets created at the formation T-junction had an aspect ratio of 1.3 (figure 5). The splitting ratios for a $Q_{\text {extra }} / Q_{\text {oil }}$ between 0 and 5 were monitored. Simulations with the $Q_{\text {extra }}=0$ were observed to split equally at first, but the splitting became unstable immediately because the daughter droplet sometimes partially entered the extra inlet, slowed down and thus destabilizing normal flow. Occasionally the droplet would break up into smaller parts as well. A small $Q_{\text {extra }}$ of $0.06 \mu \mathrm{L} / \mathrm{min}$ or $Q_{\text {extra }} / Q_{\text {oil }}=0.1$ was 
necessary to create a stable system, resulting in a split into two droplets of $51 \%$ and $49 \%$ of the original volume. Increasing the extra oil flow rate resulted in a linear increase of the volume of the largest daughter droplet in percentage of the original volume up to $Q_{\text {extra }} / Q_{o i l}=4$, where the droplets stopped splitting and the complete droplets followed the loop opposite of the extra inlet (figure 8(a)). These results are in line with those reported in literature (Yamada et al. 2008), but improve the splitting ratio up to 20:1 while keeping the droplet frequency around $1 \mathrm{~Hz}$.

(a)

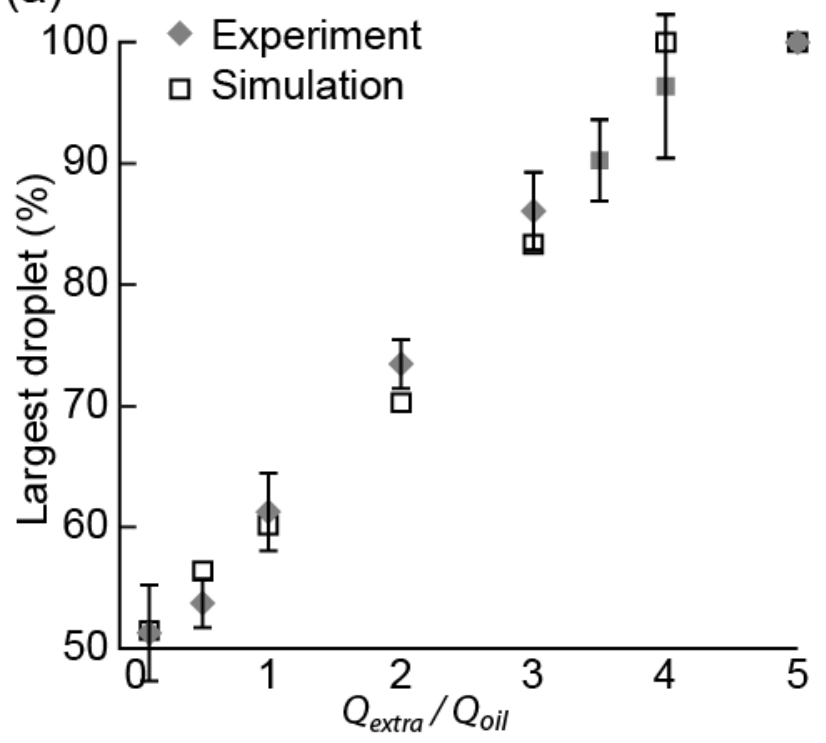

(b)

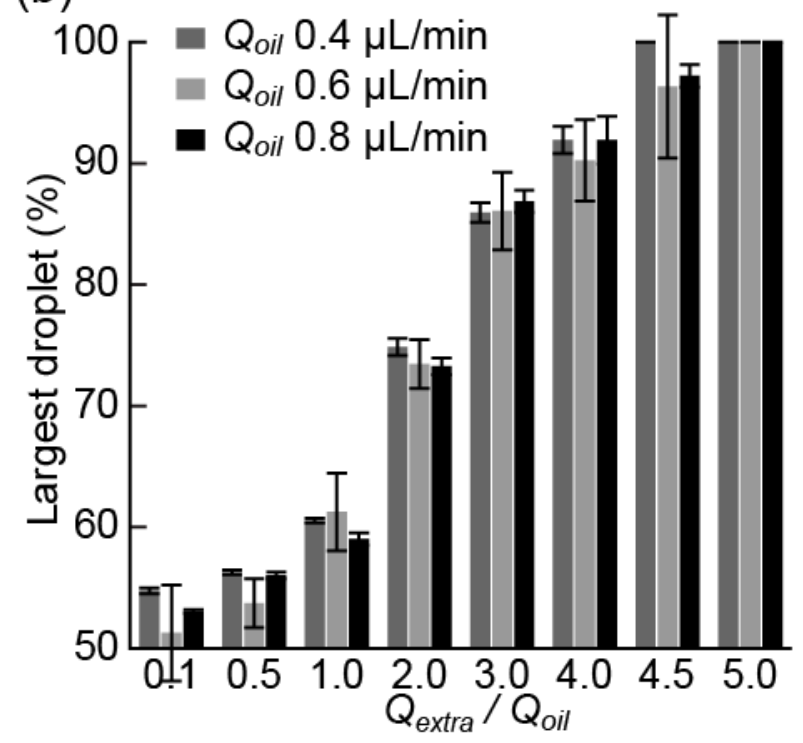

Fig 8: (a) Comparing the simulation and experimental results for droplet splitting with the extra flow. The volume of the largest daughter droplet in percentage of the original volume is plotted as a function of $Q_{\text {extra }} / Q_{o i l} ; Q_{o i l}=0.6$ $\mu \mathrm{L} / \mathrm{min}$ and $Q_{\text {water }}=0.06 \mu \mathrm{L} / \mathrm{min}$. (b) The experimental results of the volume of the largest daughter droplet in percentage of the original volume as a function of $Q_{\text {extra }} / Q_{o i l}$ for three different $Q_{o i l}: 0.4,0.6$ and $0.8 \mu \mathrm{L} / \mathrm{min}$. Error bars represent the standard deviation based on 12 consecutive droplets.

Figure 9 shows a close up from the region around the pillars on the chip which was used to calculate the aspect ratio of the droplets. For $Q_{\text {extra }} / Q_{o i l}=1$, the splitting resulted in a $57 \%-43 \%$ split (a). The smaller droplet was transported faster, due to the additional flow and was thus positioned more to the right in the image. With an increased $Q_{\text {extra }} / Q_{o i l}(2.5)$, the droplet was split in $72 \%$ and $28 \%$ (b). For higher $Q_{\text {extra }} / Q_{o i l}$ ratios (e.g. 4.0), the smallest daughters droplet were transported much faster, which made it impossible to image the two droplets in the same field of view. An overview of all results is shown in figure 8 (a). The experimental 
results correspond very well to the simulated ratios. For $Q_{\text {extra }} / Q_{o i l}=4$, the simulation predicts that the droplets follow the loop opposite of the extra inlet without splitting. However, during the experiments, most droplets still split at $Q_{\text {extra }} / Q_{o i l}=4$, although some droplets would take the opposite loop intact. The system became rather unstable at these conditions, with higher variation as result. If the extra oil flow rate was increased to $Q_{\text {extra }} / Q_{o i l}=4.5$ all droplets did take the opposite loop without splitting.

The simulations with a different $Q_{\text {oill }}$, both 0.8 and $0.4 \mu \mathrm{L} / \mathrm{min}$, but the same $Q_{\text {extra }} / Q_{\text {oil }}$ and $Q_{\text {water }} / Q_{\text {oil }}$ predicted exactly the same splitting ratios (not presented) as the simulation for $Q_{\text {oil }}=0.6 \mu \mathrm{L} / \mathrm{min}$. Figure 8 (b) compares the experimental data for these three conditions. The average volume of the largest daughter droplet in percentage of the original volume varies only slightly when the $Q_{o i l}$ is changed, showing that the droplet splitting is dependent on the $Q_{\text {extral }} / Q_{o i l}$ and not on the $Q_{\text {oil }}$. The standard deviation is mostly caused by variation in droplet formation, higher in the case of $Q_{o i l}=0.6 \mu \mathrm{L} / \mathrm{min}$, as variations in the original size lead to less stable splitting. In general the variation between different experiments is similar to the variation within one experiment.
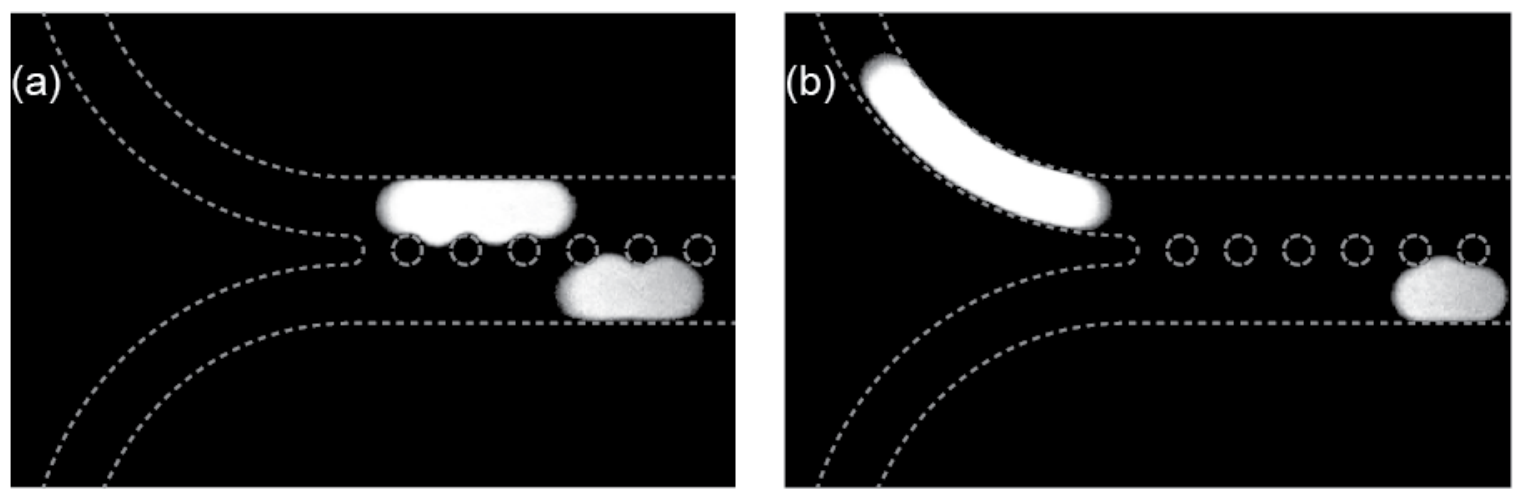

Fig 9: Close-up of the region around the pillars on the chip, the dotted line represents the outline of the channels. The smaller droplet always travels a little faster and arrives first at the pillars. (a) $Q_{\text {extra }} / Q_{o i l}=1$ resulting in a $57 \%$ - $43 \%$ split. (b) Experimental result for $Q_{e x t r a} / Q_{o i l}=2.5$, the droplet was split in $72 \%$ and $28 \%$.

\section{Conclusions}

In this article a CFD model was developed to simulate the droplet splitting system for segmented flows. In this system the splitting of the droplets was controlled by an additional oil flow. The same model was used to 
predict the working range of the operational parameters of the splitter. First the CFD model was developed and validated using droplet formation at a typical T-junction. Simulated and experimental results corresponded very well and were comparable to published results (Garstecki et al. 2006), validating the CFD model. Next the equal split design was studied using numerical simulations, leading to the narrowed Tjunction split with loop as used by Nie and Kennedy (Nie and Kennedy 2010). The flow conditions for which droplets split or do not split were determined.

Then model was extended to simulate unequal droplet splitting as well. The influence of the design parameters of an additional oil inlet to the loop was simulated, to control the pressure in the two branches of the T-junction and loop system. Three different locations and in three different layouts were compared and the impact of the location was found to be limited. The opposing inlet geometry created an unstable splitting regime. An inlet close to the T-junction and parallel to the main flow direction was chosen. In this design different operational parameters were simulated to predict the splitting ratios at varying oil flow rates in the extra inlet. Next the chosen design was fabricated in PDMS to validate the simulated results experimentally. The simulated and experimental data were found to correspond very well, suggesting the value of the CFD model to optimize this or new designs. Therefore an objective function should be formulated and optimized using the numeric model. In order to understand the influence and relevance of the multiple parameters, a dimensional analysis would be necessary, starting with channel dimensions and fluid properties.

The resulting microfluidic design was able to split droplets in two unequal parts, in a range of $51 \%-49 \%$ to $95 \%-5 \%$, independent of original droplet aspect ratio, using dynamic control. Even higher splitting ratios might possible when starting from larger original droplets. The microfluidic chip was produced by a typical SU8 mold and PDMS casting (Duffy et al. 1998), but without using the elastic properties of PDMS, such as a membrane, thus the concept is valid in other materials. No moving parts, valves, electronics or lasers were used, making the concept material independent and straightforward, ideally suited for large scale production. 


\section{Acknowledgements}

The authors thank the Flemish Institute for the Promotion of Innovation through Science and Development (IWT grant: 81166), the Flemish Fund for Scientific Research (FWO grant G0767.09 and Postdoctoral mandate Frederik Ceyssens), KU Leuven (OT project 08/023) and EU FP-7 Marie-Curie ITN-BioMax. Special thanks go to Mark Romanowski and Ralph Sperling from the Weitz lab of Harvard University who freely provided their custom perfluorinated surfactant as well as priceless advice.

\section{References}

Anna SL, Bontoux N, Stone HA (2003) Formation of dispersions using "flow focusing" in microchannels. Applied Physics Letters 82:364. doi: 10.1063/1.1537519

Atalay YT, Verboven P, Vermeir S, et al. (2008) Design optimization of an enzymatic assay in an electrokinetically-driven microfluidic device. Microfluidics and Nanofluidics 5:837-849. doi: 10.1007/s10404-008-0291-7

Atalay YT, Witters D, Vermeir S, et al. (2009) Design and optimization of a double-enzyme glucose assay in microfluidic lab-on-a-chip. Biomicrofluidics 3:44103. doi: 10.1063/1.3250304

Brackbill J, Kothe D, Zemach C (1992) A continuum method for modeling surface tension. Journal of computational physics 335354:335-354. doi: 10.1016/0021-9991(92)90240-Y

Christopher GF, Bergstein J, End NB, et al. (2009) Coalescence and splitting of confined droplets at microfluidic junctions. Lab on a chip 9:1102-9. doi: 10.1039/b813062k

Dreyfus R, Tabeling P, Willaime H (2003) Ordered and Disordered Patterns in Two-Phase Flows in Microchannels. Physical Review Letters 90:1-4. doi: 10.1103/PhysRevLett.90.144505

Duffy DC, McDonald JC, Schueller OJ, Whitesides GM (1998) Rapid Prototyping of Microfluidic Systems in Poly(dimethylsiloxane). Analytical chemistry 70:4974-84. doi: 10.1021/ac980656z

Garstecki P, Fuerstman MJ, Stone HA, Whitesides GM (2006) Formation of droplets and bubbles in a microfluidic T-junction-scaling and mechanism of break-up. Lab on a Chip 6:437-446.

Grodrian A, Metze J, Henkel T, et al. (2004) Segmented flow generation by chip reactors for highly parallelized cell cultivation. Biosensors \& bioelectronics 19:1421-8. doi: 10.1016/j.bios.2003.12.021

Günther A, Jensen KF (2006) Multiphase microfluidics: from flow characteristics to chemical and materials synthesis. Lab on a chip 6:1487-503. doi: 10.1039/b609851g

Hirt C, Nichols B (1981) Volume of fluid (VOF) method for the dynamics of free boundaries. Journal of Computational Physics 39:201-225. doi: 10.1016/0021-9991(81)90145-5

Holtze C, Rowat AC, Agresti JJ, et al. (2008) Biocompatible surfactants for water-in-fluorocarbon emulsions. Lab on a chip 8:1632-9. doi: 10.1039/b806706f 
Huebner A, Bratton D, Whyte G, et al. (2009) Static microdroplet arrays: a microfluidic device for droplet trapping, incubation and release for enzymatic and cell-based assays. Lab on a chip 9:692-8. doi: 10.1039/b813709a

Huebner A, Olguin LF, Bratton D, et al. (2008) Development of quantitative cell-based enzyme assays in microdroplets. Analytical chemistry 80:3890-6. doi: 10.1021/ac800338z

Huebner A, Srisa-Art M, Holt DJ, et al. (2007) Quantitative detection of protein expression in single cells using droplet microfluidics. Chemical communications 1218-20. doi: 10.1039/b618570c

Kolb WB, Cerro RL (1993) The motion of long bubbles in tubes of square cross section. Physics of Fluids A: Fluid Dynamics 5:1549. doi: 10.1063/1.858832

Link DR, Anna SL, Weitz DA, Stone HA (2004) Geometrically Mediated Breakup of Drops in Microfluidic Devices. Physical Review Letters 92:-. doi: 10.1103/PhysRevLett.92.054503

Liu H, Zhang Y (2011) Droplet formation in microfluidic cross-junctions. Physics of Fluids 23:082101. doi: 10.1063/1.3615643

Lombardi D, Dittrich PS (2011) Droplet microfluidics with magnetic beads: a new tool to investigate drugprotein interactions. Analytical and bioanalytical chemistry 399:347-52. doi: 10.1007/s00216-010-43027

De Lózar A, Juel A, Hazel AL (2008) The steady propagation of an air finger into a rectangular tube. Journal of Fluid Mechanics 614:173. doi: 10.1017/S0022112008003455

De Menech M, Garstecki P, Jousse F, Stone HA (2008) Transition from squeezing to dripping in a microfluidic T-shaped junction. Journal of Fluid Mechanics 595:141-161. doi: 10.1017/S002211200700910X

Nie J, Kennedy RT (2010) Sampling from nanoliter plugs via asymmetrical splitting of segmented flow. Analytical chemistry 82:7852-6. doi: 10.1021/ac101723x

Nightingale AM, De Mello JC (2010) Microscale synthesis of quantum dots. Journal of Materials Chemistry 20:8454. doi: 10.1039/c0jm01221a

Nisisako T, Torii T (2008) Microfluidic large-scale integration on a chip for mass production of monodisperse droplets and particles. Lab on a chip 8:287-93. doi: 10.1039/b713141k

Niu X, Gulati S, Edel JB, DeMello AJ (2008) Pillar-induced droplet merging in microfluidic circuits. Lab on a chip 8:1837-41. doi: 10.1039/b813325e

Olbricht WL (1996) Pore-Scale Prototypes of Multiphase Flow in Porous Media. Annual Review of Fluid Mechanics 28:187-213. doi: 10.1146/annurev.fl.28.010196.001155

Pan X, Zeng S, Zhang Q, et al. (2011) Sequential microfluidic droplet processing for rapid DNA extraction. Electrophoresis 1-7. doi: 10.1002/elps.201100078

Pekin D, Skhiri Y, Baret J-C, et al. (2011) Quantitative and sensitive detection of rare mutations using dropletbased microfluidics. Lab on a chip 11:2156-66. doi: 10.1039/c1lc20128j

Sivasamy J, Wong T-N, Nguyen N-T, Kao LT-H (2011) An investigation on the mechanism of droplet formation in a microfluidic T-junction. Microfluidics and Nanofluidics 1-10. doi: 10.1007/s10404-011-0767-8 
Song H, Ismagilov RF (2003) Millisecond kinetics on a microfluidic chip using nanoliters of reagents. Journal of the American Chemical Society 125:14613-9. doi: 10.1021/ja0354566

Song $\mathrm{H}$, Tice JD, Ismagilov RF (2003) A microfluidic system for controlling reaction networks in time. Angewandte Chemie 115:792-796. doi: 10.1002/ange.200390172

Srisa-Art M, deMello AJ, Edel JB (2010) High-Efficiency Single-Molecule Detection within Trapped Aqueous Microdroplets. The journal of physical chemistry B 114:15766-72. doi: 10.1021/jp105749t

Thorsen T, Roberts R, Arnold F, Quake SR (2001) Dynamic Pattern Formation in a Vesicle-Generating Microfluidic Device. Physical Review Letters 86:4163-4166. doi: 10.1103/PhysRevLett.86.4163

Whitesides GM (2006) The origins and the future of microfluidics. Nature 442:368-73. doi: $10.1038 /$ nature05058

Yamada M, Doi S, Maenaka H, et al. (2008) Hydrodynamic control of droplet division in bifurcating microchannel and its application to particle synthesis. Journal of colloid and interface science 321:401-7. doi: 10.1016/j.jcis.2008.01.036

Zheng B, Tice JD, Roach LS, Ismagilov RF (2004) A droplet-based, composite PDMS/glass capillary microfluidic system for evaluating protein crystallization conditions by microbatch and vapor-diffusion methods with on-chip X-ray diffraction. Angewandte Chemie 43:2508-11. doi: 10.1002/anie.200453974 